\title{
Onyx: A new heuristic bandwidth-constrained mapping of cores onto tile-based Network on Chip
}

\author{
Majid Janidarmian $^{1 \mathrm{a})}$, Ahmad Khademzadeh ${ }^{2}$, \\ and Misagh Tavanpour ${ }^{1}$ \\ ${ }^{1}$ CE Department, Islamic Azad University, Science \& Research Branch, \\ Tehran, Iran \\ ${ }^{2}$ Iran Telecom Research Center, Tehran, Iran \\ a)jani@srbiau.ac.ir
}

Abstract: Due to the ever-increasing complexity of System on Chip (SoC) design, and non-efficiency of electric bus to exchange data between IP cores in Giga scale, the Network on Chip (NoC) is presented with more flexible, scalable and reliable infra-structure. As mapping of IP cores on a given platform is one of three aspects of NoC design, with the focus on tile-based NoC architecture, we have introduced a heuristic method for mapping cores on mesh platform. Onyx ${ }^{1}$ algorithm is a method with less complexity, and it minimizes hop count between IP cores, leading to improving energy consumption and other performance parameters. We have used this method with two real applications, i.e. VOPD $^{2}$, and MPEG-4 and compared it with some existing algorithms. The results show that our developed method is more efficient.

Keywords: Network on Chip, topology, mapping, core graph, NoC architecture graph, communication cost, bandwidth constraints

Classification: Integrated circuits

\section{References}

[1] S. Kumar, A. Jantsch, J. P. Sonioen, M. Forsell, M. Millberg, J. Oeberg, K. Tiensirja, and A. Hemani, "A network on chip architecture and design methodology," IEEE Symp. VLSI ISVLSI02, pp. 105-112, Pittzburg, USA, April 2002.

[2] Umit Y. Ogras and J. Hu, "Key Research Problems in NoC Design: A Holistic Perspective," Proc. CODES+ISSS'05, New Jersey, USA, pp. 6974, Sept. 19-21, 2005.

[3] S. Murali and G. De Micheli, "Bandwidth Constrained Mapping of Cores onto NoC Architectures," Proceedings of Design, Automation and Test in Europe Conference and Exhibition (DATE), vol. 2, pp. 896-901, Feb. 2004.

[4] T. Shen, C. H. Chao, Y. K. Lien, and A.Y. Wu, "A New Binomial Mapping and Optimization Algorithm for Reduced-Complexity Mesh-based on-chip

\footnotetext{
${ }^{1}$ The stone of self mastery and self control

${ }^{2}$ Video Object Plane Decoder
} 
Network," Proceedings of the First International Symposium Networks-onChip (NOCS'07), pp.317-322, May 2007.

[5] Jingcao Hu and Radu Marculescu, "Energy-aware mapping for tile-based NoC architectures under performance constraints," Asia and South Pacific Proceedings of the ASP-DAC on Design Automation Conference, 21-24, pp. 233-239, Jan. 2003.

[6] N. Koziris et al., "An Efficient Algorithm for the Physical Mapping of Clustered Task Graphs onto Multiprocessor Architectures," Proc. of 8th EuroPDP, pp. 406-413, Jan. 2000.

[7] P. Pande, C. Grecu, A. Ivanov, and R. Saleh, "Design of a switch for network on chip applications," International Symposioum Circuits Systems, pp. 217-220, May 2003.

\section{Introduction}

The network on chip is a novel approach to overcome the constraints of SoC, and to establish inter-chip communication based on data communication network [1]. One of the influencing factors in energy consumption, latency and other performance parameters of NoC is topology. In this research, the mesh topology is selected as the platform for mapping cores due to its more flexibility as far as scalability and implementation are concerned. In this platform, each core is linked to a router by a resource network interface (RNI). Different mapping algorithms are presented to decide which core should be linked to which router. Considering the importance of mapping in $\mathrm{NoC}$ as one of three aspects of NoC design [2], in this article we attempt to present a heuristic mapping model that could map cores on the mesh platform automatically.

\section{Related Works}

The current researches mostly focus on mapping techniques for NoC platforms with two dimension mesh topology. The NMAP method that runs mapping with regard to bandwidth constraints and minimizing communication delay is selected as the criterion in most projects, and is highly efficient as far as communication cost is considered [3]. In [4] a binomial mapping method is introduced. The latter comes along with an optimal algorithm aiming at minimizing total traffic on network, the number of hops, and hardware costs. The Branch-and-Bound algorithm, presented in [5] has been able to map IP cores on tile-based NoC architecture, and it has tried to minimize total energy consumption, and to overcome bandwidth constraints. Also, the mapping of clusters onto the physical topology of processors has been studied in the field of parallel processing. In [6], PMAP, a two-phase mapping algorithm for placing clusters onto processors is presented. In this field, the mappings produced by the PMAP algorithm are shown to have lower communication costs than mappings with previous algorithms.

\section{Mathematical Formulation of Mapping}

Each application is divided to several IP cores. The manner of relation 
between cores is shown by a core graph.

Definition1: The core graph is a directional graph $\mathrm{G}(\mathrm{V}, \mathrm{E})$, whose each vertex $\mathrm{v}_{\mathrm{i}} \in \mathrm{V}$ shows a core, and a directional edge $\mathrm{e}_{\mathrm{i}, \mathrm{j}} \in \mathrm{E}$ illustrates connection between $v_{i}$ and $v_{j}$. The weight of $e_{i, j}$ that is shown as comm $\mathrm{i}_{\mathrm{i}, \mathrm{j}}$, represents the bandwidth requirement of the communication from $v_{i}$ to $v_{j}$.

We display an IP core along with a router connected to it by a RNI as a tile, and the manner of tiles' link and bandwidth between tiles would be determined by NoC architecture graph.

Definition2: The NoC architecture graph is a directional graph $\mathrm{A}(\mathrm{T}, \mathrm{L})$ whose each vertex $t_{i} \in \mathrm{T}$ represents a tile in the NoC architecture, and its directional edge that is shown by $\mathrm{l}_{\mathrm{i}, \mathrm{j}} \in \mathrm{L}$ shows a physical link from $\mathrm{t}_{\mathrm{i}}$ to $\mathrm{t}_{\mathrm{j}}$ and for each $l_{i, j}$ a $B W\left(l_{i, j}\right)$ is considered. In this paper, tiles are connected to each other through a mesh interconnection. We have employed $x-y$ routing algorithm and $r_{i, j}$ shows routing path between $t_{i}$ and $t_{j}$. Each $r_{i, j}$ includes $a$ number of links out of existing links in NoC architecture, and these links are defined with $\mathrm{L}\left(\mathrm{r}_{\mathrm{i}, \mathrm{j}}\right)$. The definitions are presented in Fig. 1-a.

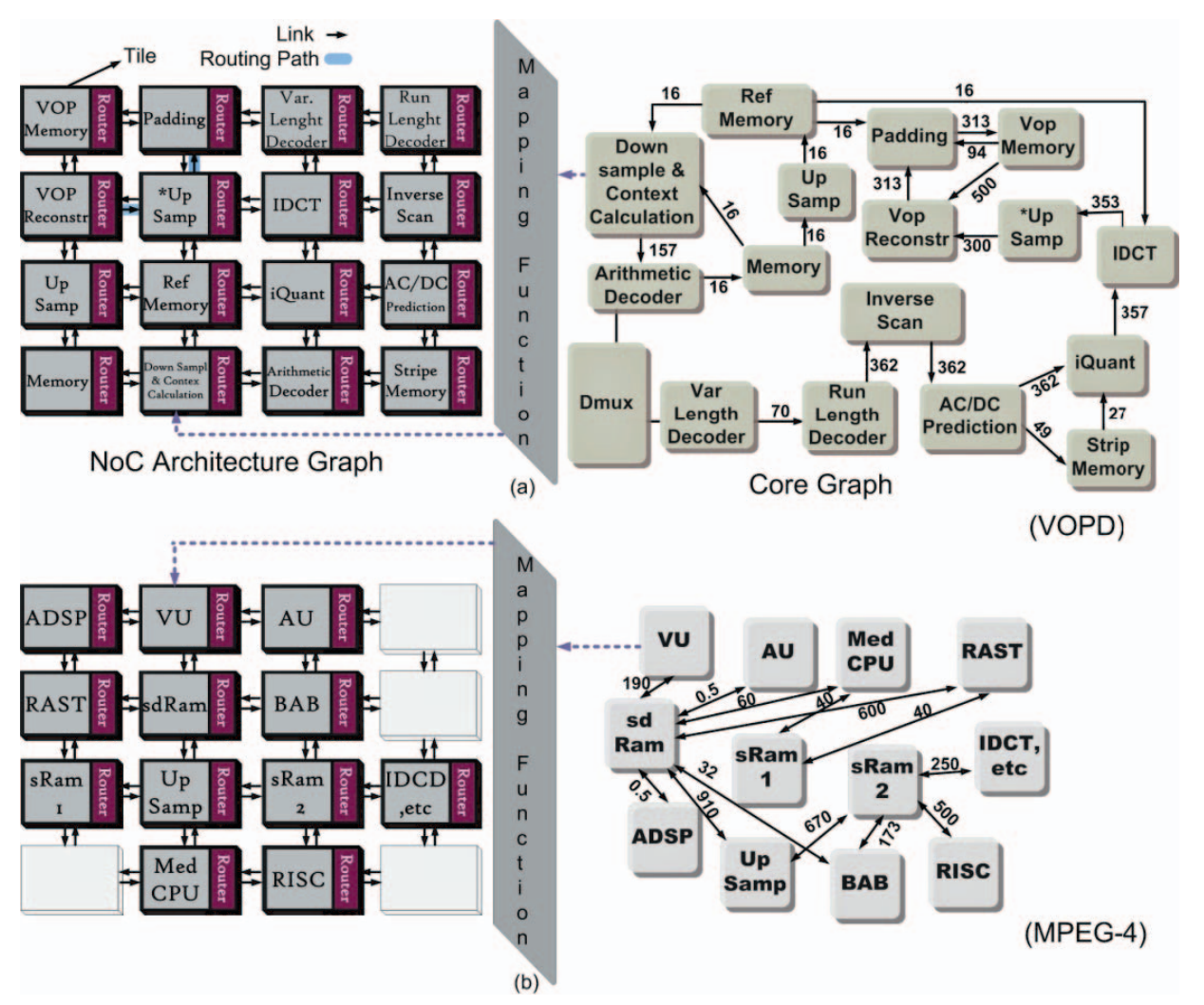

Fig. 1. (a) Mapping of VOPD core graph on NoC Architecture graph, (b) Mapping of MPEG-4 core graph on NoC Architecture graph

The core graph mapping $G(V, E)$ on NoC architecture graph $A(T, L)$ is defined by a one to one mapping function.

$$
\text { map : } \mathrm{V} \rightarrow \mathrm{T} \text {, s.t. } \operatorname{map}\left(\mathrm{v}_{\mathrm{i}}\right)=\mathrm{t}_{\mathrm{j}}, \forall \mathrm{v}_{\mathrm{i}} \in \mathrm{V}, \exists \mathrm{t}_{\mathrm{j}} \in T,|V| \leq|T|
$$


sented as $\mathrm{d}^{\mathrm{k}}$ and its amount which indicates the required bandwidth for each edge is displayed with $\mathrm{vl}\left(\mathrm{d}^{\mathrm{k}}\right)$. The set of all commodities represented as D is achieved as follows:

$$
D=\left\{\begin{array}{l}
\mathrm{d}^{\mathrm{k}}: \operatorname{vl}\left(\mathrm{d}^{\mathrm{k}}\right)=\operatorname{comm}_{\mathrm{i}, \mathrm{j}}, \mathrm{k}=1,2, \ldots|\mathrm{E}|, \forall \mathrm{e}_{\mathrm{i}, \mathrm{j}} \in \mathrm{E}, \\
\operatorname{with} \operatorname{source}\left(\mathrm{d}^{\mathrm{k}}\right)=\operatorname{map}\left(\mathrm{v}_{\mathrm{i}}\right), \operatorname{dest}\left(\mathrm{d}^{\mathrm{k}}\right)=\operatorname{map}\left(\mathrm{v}_{\mathrm{j}}\right)
\end{array}\right\}
$$

The BW constraints are displayed using by inequality:

$$
\begin{aligned}
& \forall \text { link } l_{n, m}, \sum_{k=1}^{|\mathrm{E}|} \mathrm{vl}\left(\mathrm{d}^{\mathrm{k}}\right) \times \mathrm{f}\left(\mathrm{l}_{\mathrm{n}, \mathrm{m}}, \mathrm{r}_{\text {source }\left(\mathrm{d}^{\mathrm{k}}\right), \operatorname{dest}\left(\mathrm{d}^{\mathrm{k}}\right)}\right) \leq B W\left(\mathrm{l}_{\mathrm{n}, \mathrm{m}}\right) \\
& \mathrm{f}\left(\mathrm{l}_{\mathrm{n}, \mathrm{m}}, \mathrm{r}_{\mathrm{i}, \mathrm{j}}\right)=\left\{\begin{array}{c}
1, \text { if } l_{n, m} \in L\left(r_{i, j}\right) \\
0, \text { otherwise }
\end{array}\right\}
\end{aligned}
$$

Considering the above-mentioned issues, it can be stated that a proper mapping should overcome bandwidth constraints, and improve the energy consumption and other performance parameters too. To calculate energy consumption a model is introduced in [7]. This formula is designed based on energy for sending one bit on communication channel from node $\mathrm{i}$ to node $\mathrm{j}$ in mesh topology, as illustrated in Formula 5. $E_{b i t}^{t i, t j}$ indicates energy consumption of transmission of one bit between two tiles. $E_{R b i t}$ and $E_{l b i t}$ display energy consumption to forward one bit in router and link respectively. $n_{\text {hops }}$ is the number of routers that one bit has to pass to be sent from the source tile to the destination one. And finally energy consumption for transmitted data could be displayed by Formula 6 :

$$
\begin{aligned}
E_{b i t}^{t i, t j} & =\sum_{\mathrm{k}=1}^{n_{\text {hops }}} \mathrm{E}_{\mathrm{R}_{\mathrm{k}} \mathrm{bit}}+\left(n_{\text {hops }}-1\right) \times E_{\text {lbit }} \\
E_{\text {total }}^{t i, t j} & =\text { Data size } \times E_{\text {bit }}^{t i, t j}
\end{aligned}
$$

As illustrated above, one significant approach to lessen energy consumption is to limit $n_{\text {hops }}$ between related cores in core graph as much as possible. It goes without saying that the very approach would be effective for other performance parameters including latency.

\section{Onyx Mapping Algorithm}

We aim at mapping each IP core of core graph on a tile so that a connection between tiles is established by the existing router in each tile. We have employed so-called Onyx, a heuristic method with little complexity and best results for mapping. As initially there is no mapping on NoC architecture tiles, and with cores mapping the possibility of better mapping would be decreased, cores with higher $\operatorname{comm}_{i, j}$ should be mapped earlier. At first a core with the highest priority for mapping is determined by the function highest-priority $(G(V, E))$. Then the function finds the highest $\operatorname{comm}_{i, j}$, and between the source core and its edge destination, the core with higher $\operatorname{ranking}\left(C_{i}\right)$ will be selected as the first choice. After selecting the first core 
for mapping, a proper location on mesh based NoC architecture should be selected, as it can influence mapping procedure greatly.

$$
\operatorname{Ranking}\left(C_{i}\right)=\sum_{\substack{\forall, 2, \ldots|V| \\ i \neq j}}\left(\operatorname{comm}_{i, j}+\operatorname{comm}_{j, i}\right)
$$

In mesh platform, with respect to the number of neighboring tiles, there are three types of tiles. Each tile, considering its location in the mesh platform may have 2, 3, or 4 neighbors. Hence, an appropriate router of each tile can be used resulting in $3 \times 3,4 \times 4$ or $5 \times 5$ (Fig. 2-a). Each tile may have different distances with every one of above-mentioned tiles. Considering mesh platform as a symmetrical platform, it is observed that in $\mathrm{n} \times \mathrm{n}$ mesh only
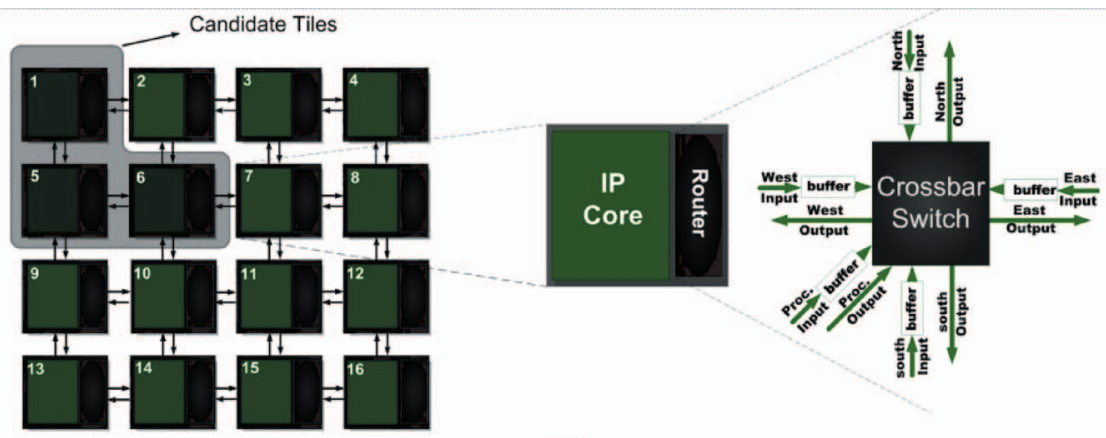

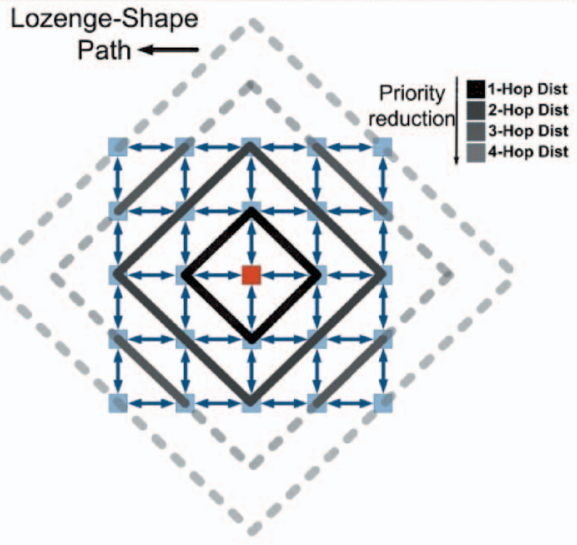

(b)

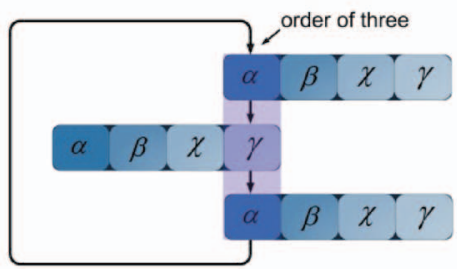

(d) (a)

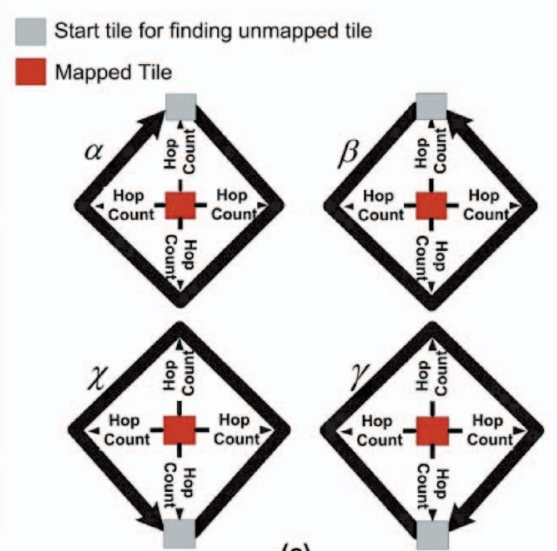

(c)

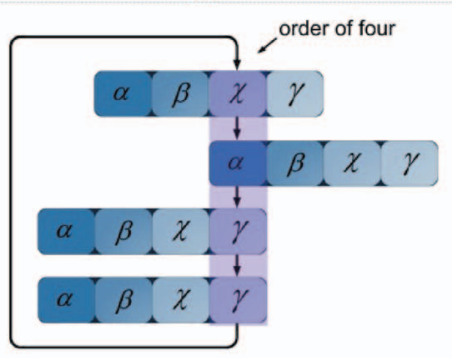

Fig. 2. (a) Candidate tiles for mapping the first core, (b) Concept of Lozenge-shape Path, (c) Definition of 4 Movements for moving on lozenge-shape path to determine priority of tiles for mapping, (d) A sample of selection of threes or fours order using 4 movements 
$\sum_{i=1}^{\left\lceil\frac{n}{2}\right\rceil}$ i different tiles can be selected as the candidate for the location of first core. As other tiles have similar condition with one of this collection's tiles, the first core should be put on one of the candidate locations, and finally the most appropriate location will be determined. As represented by Fig. 2-a, onyx has selected the candidate tiles for mapping the first core.

As stated earlier, to get high performance, cores of the core graph which are tight related should be more adjacent on NoC architecture graph, and we attempt to reach such outcome in this research. To do so, while studying mesh platform as the communication infrastructure, it can be seen that as far as one tile in this NoC architecture concerned, tiles which have the same distance with the mentioned tile form a lozenge-shape path (Fig. 2-b). Following such method can decrease algorithm complexity and its execution speed, because if a core is going to be put in the nearest possible distance with its related core, first in the lozenge-shape path it looks for a unmapped tile with one hop and if it did not succeed it goes ahead to the two hops distance, and this mechanism continues until the empty tile is identified. Onyx goes along with 4 types of movement to determine the movement direction on lozenge-shape path (Fig. 2-c), and these types of movement also determine the tiles priority for mapping. In fact, relying on these 4 types of movement, it selects an order of three or four and follows the same order to the mapping end. To move on the lozenge-shape path it uses a movement on the line, and after core mapping it is the turn of next movement (Fig. 2-d). Onyx algorithm uses different, however limited orders of three or four with the help of 4 movements to achieve the best result. Therefore, after mapping the first core in one of the candidate locations, out of IP cores in core graph which are waiting for mapping, a core should be selected that has the highest comm with the mapped core, and then it should be placed in the shortest distance with it. Finding a location with such feature in terms of lozenge-shape path and priority and movement direction on it using an order of three or four would be easy. This procedure continues until mapping of all cores. More details are supplied in the following pseudo code (Fig. 3-a). The presented algorithm with focus on decreasing the number of hops between related cores can decrease commcost, and performance would rise consequently. The commcost is calculated as Formula 8:

$$
\text { commcost }=\sum_{k=1}^{|\mathrm{E}|} \operatorname{vl}\left(\mathrm{d}^{\mathrm{k}}\right) \times \operatorname{dist}\left(\operatorname{source}\left(\mathrm{d}^{\mathrm{k}}\right), \operatorname{dest}\left(\mathrm{d}^{\mathrm{k}}\right)\right)
$$

\section{Experiment Results}

We have used VOPD and MPEG-4 real core graph to compare Onyx algorithm with other algorithms. For mapping VOPD, the order of $\alpha-\gamma-\alpha$ movements has been the best movement order on lozenge-shape path, and tile 5 is the best candidate for first mapping. For mapping MPEG- 4 , the order $\chi-\alpha-\gamma-\gamma$ and tile 6 have had the best condition. The movement orders are shown in Fig. 2-d. The ultimate results of mapping Onyx on core graphs VOPD and MPEG-4 are presented in Fig. 1. We have compared 


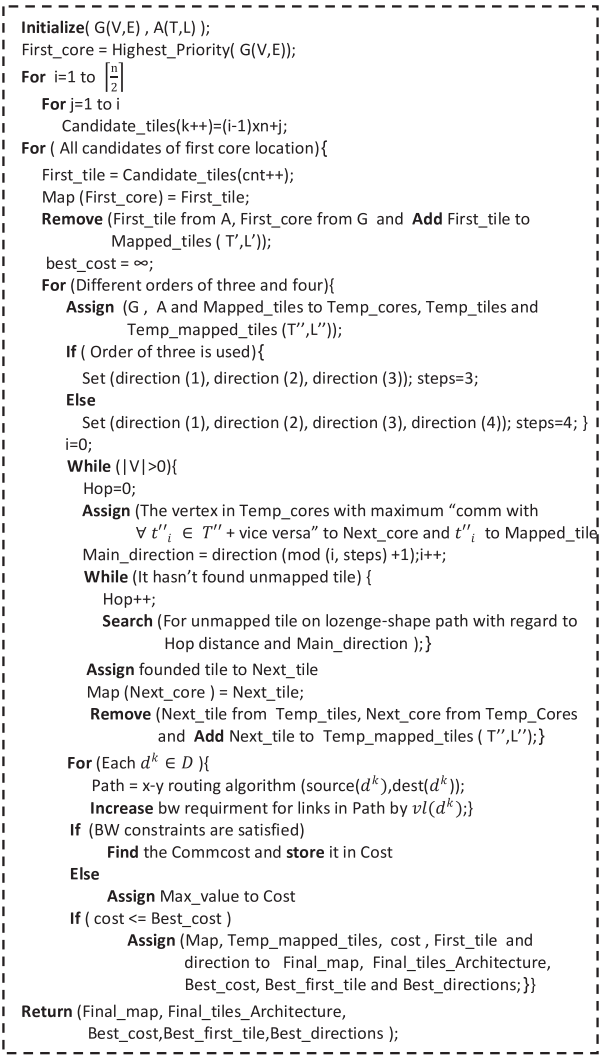

(a)

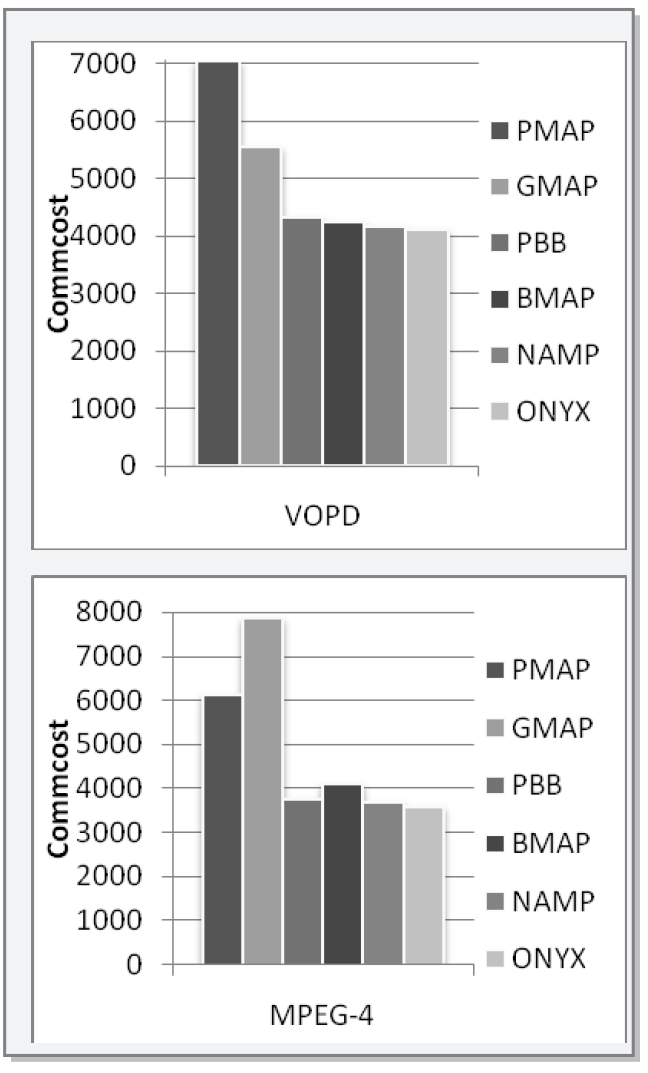

(b)

Fig. 3. (a) pseudo code of Onyx mapping algorithm, (b) Communication Cost of Different Mapping Algorithms on VOPD and MPEG-4 Core Graph

Onyx with NMAP, Partial Branch-and-Bound, GMAP, PMAP in [3] and BMAP [4]. In Fig. 3-b the minimum communication costs with the same bandwidth constraints for all algorithms are presented. As illustrated, our developed algorithm is showing best results.

\section{Conclusion and Future Work}

In this paper, a heuristic method for mapping the cores onto a tile-based NoC architecture was proposed in the way that tiles were connected to each other through a mesh interconnection. Onyx, defines four movements to assign priorities to the tiles on a lozenge-shape path, and appears to obtain better communication cost compared with a number of efficient mapping algorithms. Reducing the hop count between the directly connected cores in the core graph has a huge effect on the process of optimizing performance parameters such as energy consumption and latency. Onyx could obtain this goal, and seems to offer the most efficient method in reducing the hop count between the adjacent cores. The idea of implementing Onyx on other topologies can be an interesting subject for the further studies. Furthermore, since mapping is one of the most important sections in the process of designing NoCs, developing new optimization ideas to be used during the mapping procedure, could possibly lead us to much better results.

\section{(C) IEICE 2009}

DOI: $10.1587 /$ elex.6.1 Received September 10, 2008 Accepted November 17, 2008 Published January 10, 2009 